\title{
miR-137 suppresses proliferation, migration and invasion of colon cancer cell lines by targeting TCF4
}

\author{
WEI-PING BI ${ }^{1}$, MIN XIA $^{2}$ and XIN-JIAN WANG ${ }^{3}$ \\ Departments of ${ }^{1}$ Gastroenterology, ${ }^{2}$ Endoscopy, and ${ }^{3}$ General Surgery, \\ Wendeng Central Hospital of Weihai City, Weihai, Shandong 264400, P.R. China
}

Received November 3, 2015; Accepted May 23, 2017

DOI: $10.3892 / \mathrm{ol} .2018 .8364$

\begin{abstract}
Colorectal cancer is cancer of the colon or rectum and is the third most prevalent form of cancer. Currently, there are several shortcomings in the prognosis and early detection of colon cancer. The present study aims to address questions pertaining to the role of microRNA (miR)-137 in colon cancer progression and the mode of regulation. The endogenous and over-expressed levels of miR-137 in three colon cancer cell lines were assessed by reverse transcription-quantitative polymerase chain reaction (RT-qPCR). The MTT assay was used to assess cell proliferation. Cell migration and invasion assays were assessed using Transwell apparatus and Matrigel invasion chambers. The potential targets of miR-150 were predicted using TargetScan software, and one of the best scoring targets, transcription factor 4 (TCF4), was experimentally validated using western blot analysis and RT-qPCR. It was found that that miR-137 is expressed at extremely low levels in COLO205, HCT116 and SW480 cell lines. Cell proliferation, migration and invasion were inhibited subsequent to transfection of the colon cancer cell lines with miR-137. Using bioinformatics analysis, the best scoring putative targets were identified. One such target, TCF4, was experimentally validated, and it was shown that overexpression of miR-137 suppresses TCF4 in all three colon cancer cell lines. In conclusion, it was shown that miR-137 inhibits cell proliferation, migration and invasion in colon cancer cell lines by negatively regulating the expression of TCF4.
\end{abstract}

\section{Introduction}

Colorectal cancer is cancer of the colon or rectum, with $\sim 1,000,000$ cases reported in 2012 (1). It is the third most prevalent from of cancer. The American Cancer Society estimates 135,430 individuals are newly diagnosed with colorectal

Correspondence to: Dr Xin-Jian Wang, Department of General Surgery, Wendeng Central Hospital of Weihai City, 3 West Mishan East Road, Wendeng, Weihai, Shandong 264400, P.R. China

E-mail: wangxinjianxj@outlook.com

Key words: miR-137, colon cancer, TCF4, migration, invasion cancer with 50,260 deaths in 2017 (1), making it a significant health burden that requires immediate attention. Currently, the treatment methods for colon cancer involve various therapies, notably chemotherapy and radiation therapy. However, for improved prognosis and early detection, the underlying mechanisms of colon cancer progression and metastasis require elucidation (2).

Accumulating evidence in the past decade has established an association between microRNAs (miRNAs/miRs) and cancer (3-5). miRNAs are short non-coding RNAs of 22-24 nucleotides that are involved in cellular growth, differentiation, regulation and apoptosis (3). Often deregulated, miRNAs have been shown to be involved in the progression of different types of cancer (6).

Several studies have revealed that the miRNA expression patterns are altered in colorectal cancer (7-10). One of the earliest studies that identified a correlation between miRNA expression patterns and colorectal cancer found deregulation of miR-143 and miR-145 in colorectal cancer, suggesting a tumor suppressor role of these miRNAs (7). Subsequently, a variety of miRNAs were identified that either play an oncogenic or a tumor suppressor role in colorectal cancer. For example, miR-21 has been shown to play an oncogenic role in colorectal cancer $(8,9)$. Several studies have shown altered expression levels of miR-17-92 cluster, miR-31, miR-224 and miR-183 in colorectal cancer (10). Identification of altered expression of miRNAs in colorectal cancer is important, as it may act as an early detection marker and therapeutic targets in colorectal cancer progression and treatment.

The present study investigates one such miRNA, miR-137, which has been frequently associated with different types of cancer. miR-137 has also been shown to be downregulated in gastric cancer and negatively regulates cell division cycle 42 (Cdc42) (11). A previous study demonstrated that ectopic expression of miR-137 in lung cancer cells downregulated Cdc42 and cyclin dependent kinase 6 (12). Similarly, ectopic expression of miR-137 in breast cancer cells downregulated estrogen-related receptor $\alpha$ expression at both mRNA and protein levels (13). However, the association between miR-137 and colon cancer is poorly understood. At present, there have been a small number of studies that found an association between miR-137 and colorectal cancer. In one such study, the expression of miR-137 in colorectal cell lines was inversely correlated with Cdc42 expression (14). In another similar study, 
miR-137 was found to act as a tumor suppressor in the colon by targeting lysine-specific demethylase 1 (15). However, other targets of miR-137 that regulate colon cancer cell migration and invasion remain to be identified and substantiated. The present study attempted to investigate the role of miR-137 in colon cancer progression, the downstream targets and the mode of regulation.

\section{Materials and methods}

Cell lines, cell culture and transfection. The colon cancer COLO205, HCT116 and SW480 cell lines were purchased from Shanghai Cell Bank, Chinese Academy of Sciences (Shanghai, China). The cell lines were maintained and cultured according to the manufacturer's instructions. Dulbecco's modified Eagle's medium (Thermo Fisher Scientific, Inc., Waltham, MA, USA) supplemented with $10 \%$ fetal bovine serum (Thermo Fisher Scientific, Inc.) was used for cell culture. All cell lines were cultured in the aforementioned media, and incubated at $37^{\circ} \mathrm{C}$ in a $5 \% \mathrm{CO}_{2}$ humidified incubator. Lipofectamine 2000 (Invitrogen; Thermo Fisher Scientific, Inc., Waltham, MA, USA) was used for all transfection experiments, and the transfections were performed according to the manufacturer's protocol. Cells were transfected with miR-137 (sequence, UUAUUG CUUAAGAAUACGCGUAG; Sigma-Aldrich; Merck KGaA, Darmstadt, Germany). The negative control was a validated random sequence known to exhibit no effects on miRNA function, purchased from Thermo Fisher Scientific, Inc., (cat. no. 4464058).

Cell proliferation, migration and invasion assays. The MTT method was used to determine cell proliferation. The cells were transfected with miR-137 mimics or controls and were seeded onto 96 -well plates $\left(4 \times 10^{5}\right.$ cells/well). Cell viability was documented at regular intervals, every $24 \mathrm{~h}$ for 6 days, according to the manufacturer's protocol. Formazan crystals were dissolved in dimethylsulfoxide (Sigma-Aldrich; Merck KGaA). Subsequently, $25 \mu \mathrm{l}$ of MTT solution was added to each well, followed by incubation at $37^{\circ} \mathrm{C}$ for $6 \mathrm{~h}$. The plates were centrifuged at $1,000 \mathrm{xg}$, and the absorbance of the formazan product was quantified at $490 \mathrm{~nm}$ in an ELISA reader (Bio-Rad Laboratories, Inc., Hercules, CA, USA). Cell migration was assessed in two 24 -well $8-\mu \mathrm{m}$ pore Transwell plates (BD Biosciences Franklin Lakes, NJ, USA), according to the manufacturer's instructions. The plates were then incubated for $16 \mathrm{~h}$ at $37^{\circ} \mathrm{C}$ in $5 \% \mathrm{CO}_{2}$ humidified incubator. Cell invasion was assessed using the Transwell apparatus. For the invasion assay, $\sim 1 \times 10^{6}$ cells were added to Matrigel Invasion Chambers in two 24 -well $8-\mu \mathrm{m}$ pore plates (BD Biosciences), according to the manufacturer's protocol. Following addition, the plates were incubated at $37^{\circ} \mathrm{C}$ for $36 \mathrm{~h}$ in $5 \% \mathrm{CO}_{2}$ in a humidified incubator. The cells on the upper surface of the membrane were gently scraped off subsequent to incubation. Cells that migrated to the bottom surface were fixed in $100 \%$ methanol for $5 \mathrm{~min}$ and stained with $0.4 \%$ crystal violet staining solution for $2 \mathrm{~min}$. The cells were counted from different microscopic fields with a light microscope at x300 magnification, and the values were averaged.
Table I. Sequences of primers used in the present study.

\begin{tabular}{lc}
\hline Primer & Sequence \\
\hline$\beta$-actin & \\
Forward & 5'-GGGACCTGACTGACTACCTCA-3' \\
Reverse & 5'-TGACTCGTCATACTCCTGCTTG-3' \\
GAPDH & \\
Forward & 5'-TGCACCACCAACTGCTTAGC-3' \\
Reverse & 5'-GGCATGGACTGTGGTCATGAG-3' \\
TCF4 & \\
Forward & 5'-GCAAGTTGGACGCCCGCAAGATC-3' \\
Reverse & 5'-TAGTCAGCCATGGGGCGGAGA-3'
\end{tabular}

$\mathrm{TCF}$, transcription factor.

Reverse transcription-quantitative polymerase chain reaction $(R T-q P C R)$. A kit-based approach was used to isolate the total RNA. Using RNeasy kit (Qiagen, Inc., Valencia, CA, USA), total RNA from the cells was extracted according to the manufacturer's protocol at $42^{\circ} \mathrm{C}$ for $30 \mathrm{~min}$. The final volume of the PCR mixture was $50 \mu \mathrm{l}$, and it contained $0.3 \mu \mathrm{M}$ probe, $0.3 \mu \mathrm{M}$ of forward and reverse primers, 1X PCR buffer, $0.4 \mathrm{mM}$ dNTPs and Taq DNA polymerase, all purchased from New England BioLabs, Inc. (Ipswich, MA, USA). The thermocycling conditions included initial denaturation at $95^{\circ} \mathrm{C}$ for $5 \mathrm{~min}$, followed by 35 cycles of $95^{\circ} \mathrm{C}$ for $20 \mathrm{sec}, 58^{\circ} \mathrm{C}$ for $30 \mathrm{sec}$ and $68^{\circ} \mathrm{C}$ for $40 \mathrm{sec}$, with a final extension step of $72^{\circ} \mathrm{C}$ for $10 \mathrm{~min}$. Specific RNA transcripts were quantified by SYBR-Green qPCR (Thermo Fisher Scientific, Inc.). Reactions were performed and analyzed using an ABI Prism 7700 Sequence Detection System (Applied Biosystems; Thermo Fisher Scientific, Inc.). $\beta$-actin mRNA (Macrogen, Inc., Seoul, Republic of Korea) or GAPDH mRNA (Macrogen, Inc.) was used as an internal control. Sequences of primers are presented in Table I. Data was quantified using ImageJ software (version 1.47; National Institutes of Health, Bethesda, MD, USA).

Bioinformatical analysis. TargetScan (www.targetscan.org) is a webserver prediction tool that has been routinely used to predict the biological targets of miRNAs with improved accuracy compared to other prediction programs. To identify the biological target of miR-137, miR-137 was run in TargetScan. From the top 10 targets, TCF4 was finalized based on a combination of Aggregate $\mathrm{P}_{\mathrm{CT}}$, context scores and based on whether or not miR-137 and the mRNA are expressed in cells of interest.

Western blot analysis. The proteins were extracted by lysing the cells in sample loading buffer. The composition of the loading buffer was $1.5 \%$ SDS, $10 \%$ glycerol, $5 \mathrm{mM}$ $\beta$-mercaptoethanol, bromphenol blue and $75 \mathrm{mM}$ Tris ( $\mathrm{pH} 7)$. Whole cell lysates were separated by SDS-PAGE (12\% gel) and the proteins were transferred onto a polyvinylidene fluoride membrane. Subsequently, the membranes were incubated with rabbit anti-TCF4 (GTX54531; 1:200; GeneTex International Corporation, Hsinchu, Taiwan) and anti- $\beta$-actin 
A

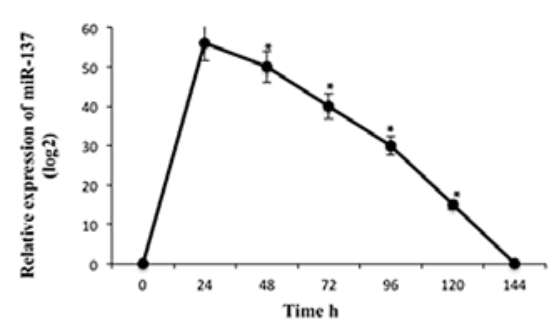

B

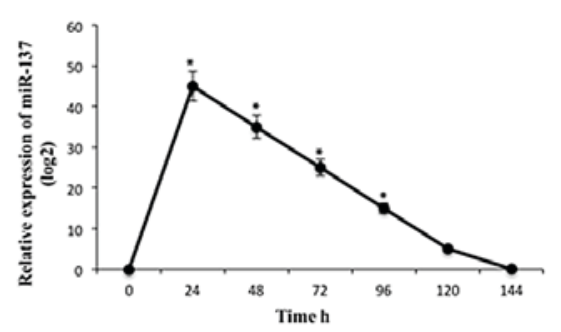

C

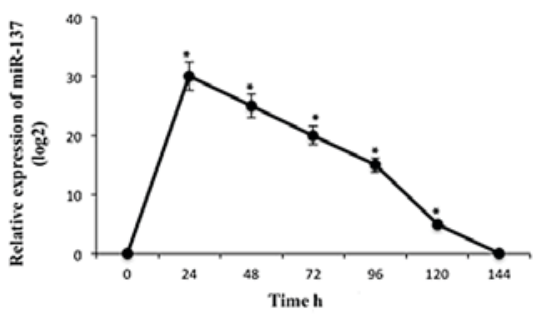

Figure 1. Ectopic expression of miR-137 in colon cancer (A) COLO205, (B) HCT116 and (C) SW480 cell lines. The levels of miR-137 were highest after the first $24 \mathrm{~h}$ period in all the cell lines and gradually decreased between 24 and $144 \mathrm{~h}$. miR-137, microRNA-137. ${ }^{*} \mathrm{P}<0.05$.

primary antibodies (cat. no. GTX109639; 1:500; GeneTex International Corporation) at $4^{\circ} \mathrm{C}$ overnight. Subsequent to incubation, horseradish peroxidase-conjugated rat anti-mouse IgG1 secondary antibodies (cat. no. GTX83207; 1:500; GeneTex, Hsinchu City, Taiwan) were used to detect specific bands.

Statistical analysis. For statistical analysis, SigmaPlot (version 13; SysStat Software, Inc., San Jose CA, USA) and Microsoft Excel (version 15.33; Microsoft Corporation, Redmond, WA, USA) were used. Student's t-test was used to determine significant differences between groups. In all statistical analyses, two sided tests were applied. $\mathrm{P}<0.05$ was considered to indicate a statistically significant difference. All data are shown as the mean \pm standard deviation.

\section{Results}

Ectopic expression of miR-137 in colon cancer COLO205, HCT116 and SW480 cell lines. Firstly, the endogenous levels of miR-137 in colon cancer COLO205, HCT116 and SW480 cell lines were assessed by RT-qPCR. The levels of miR-137 in these cell lines were low (data not shown). Following the transfection of miR-137, the expression levels of miR-137 in the cell lines were monitored at time intervals of $24 \mathrm{~h}$. The levels of miR-137 were highest following the first $24 \mathrm{~h}$ period in all the cell lines. However, the expression levels of miR-137 gradually decreased between 24 and 144 h (Fig. 1).

miR-137 suppresses cell proliferation in colon cancer cell lines. Subsequently, the effect of miR-137 on cell proliferation following transfections in all cell lines was investigated. Based on the results from the MTT assay, overexpression of miR-137 in the cell lines significantly suppressed cell proliferation $(\mathrm{P}=0.009, \mathrm{P}=0.007$ and $\mathrm{P}=0.01$; Fig. 2$)$. Analysis of the results indicated that the inhibition rate of miR-137 in colon cancer COLO205, HCT116 and SW480 cell lines was 35.34 \pm 3.46 , $29.78 \pm 4.82$ and $32.9 \pm 2.8 \%$, respectively. Overall, these results suggest that miR-137 suppresses cell proliferation in colon cancer cell lines.

miR-137 suppresses migration and invasion in colon cancer COLO205, HCT116 and SW480 cell lines. The role of miR-137 in the progression and metastasis of colon cancer was then investigated using migration and invasion assays performed with Transwell apparatus. The transfected cells from log-phase growth were cultured on the Transwell apparatus. The cells were incubated for $12 \mathrm{~h}$ for migration analysis. Analysis of the results revealed that the cell migration was significantly decreased in miR-137-transfected cells compared with corresponding negative controls $(\mathrm{P}=0.005$; Fig. 3). The effect of miR-137 on cell invasiveness was then determined by incubating the miR-137-transfected cells for $24 \mathrm{~h}$ and monitoring the cellular movement through the extracellular matrix. The results of the invasion assay indicated that miR-137-transfected cells exhibited a significantly reduced invasiveness with respect to their corresponding controls $(\mathrm{P}=0.007$; Fig. 3). Combining the analyses from the migration and invasion assays, it is clear that miR-137 suppresses migration and invasion in COLO205, HCT116 and SW480 colon cancer cell lines.

Transcription factor 4 (TCF4) is a putative target of miR-137. Subsequently, the downstream targets of miR-150 were identified to obtain information on the regulatory mechanisms. The potential targets of miR-150 were predicted using TargetScan bioinformatics software. Initial runs provided a wide range of targets regulated by miR-137; however, a more specific range of best scoring targets was selected using stringent filters; the predicted targets were shortlisted based on the efficacy of targeting or the probability of conserved targeting. Among the best scoring targets, TCF4 appeared to be of particular interest, since it has been implicated as a direct target of different miRNAs in various types of cancer, as reported by previously published studies (16-18). Therefore, experimental validation of TCF4 as a direct target of miR-137 was performed in colon cancer cell lines.

miR-137 negatively regulates TCF4 expression in colon cancer COLO205, HCT116 and SW480 cell lines. To experimentally validate the association between miR-137 and the predicted target TCF4, miR-137 was transfected into the colon cancer cell lines followed by subsequent incubation for $36 \mathrm{~h}$. The levels of TCF4 mRNA and protein expression were detected and quantified by RT-qPCR and western blot analysis. The results were analyzed, and as shown in Fig. 4, overexpression of miR-137 significantly suppressed TCF4 in all the colon cancer cell lines compared with the controls $(\mathrm{P}<0.05$; Fig. 4). This data clearly indicates that TCF4 is a direct target of miR-137 in colon cancer cell lines and overexpression of miR-137 may negatively regulate TCF4 expression in colon cancer cell lines. 
A

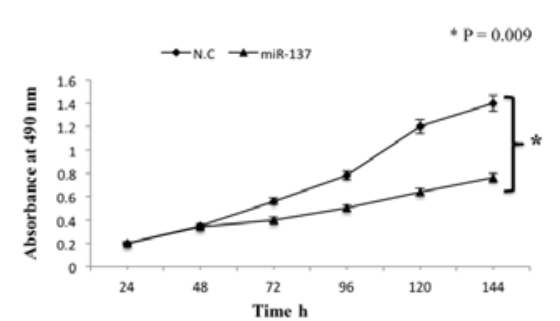

B

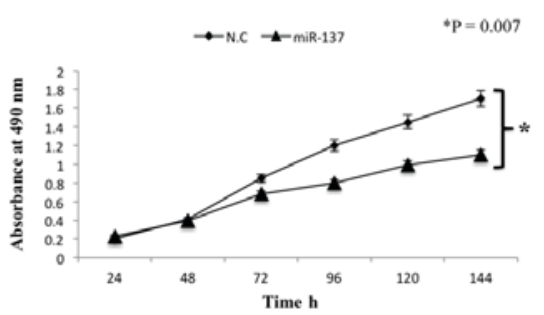

C

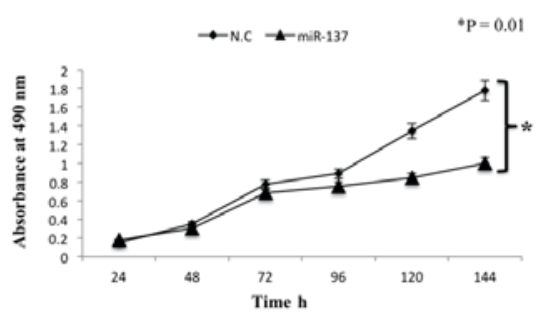

Figure 2. Cell viability of colon cancer (A) COLO205, (B) HCT116 and (C) SW480 cell lines following transfection with miR-137. Cell viability was assessed based on the results from the MTT assay, and overexpression of miR-137 in all the three cell lines. NC, negative control; miR-137, microRNA-137.
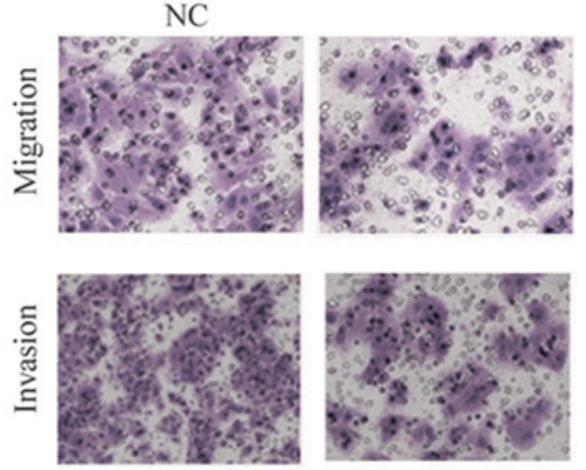

COLO205
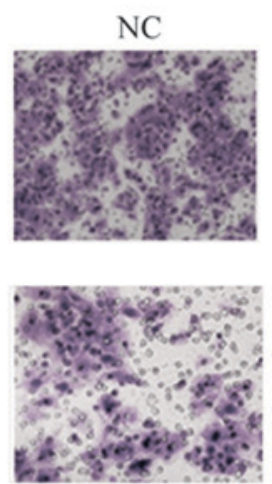

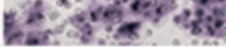

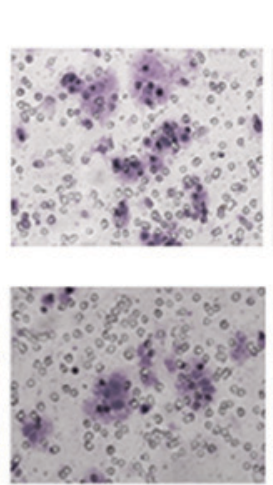

HCT116
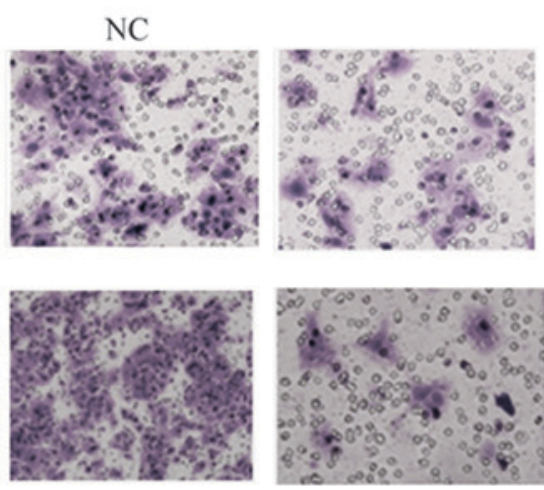

SW480

Figure 3. miR-137 suppresses migration and invasion in COLO205, HCT116 and SW480 colon cancer cell lines following transfection with miR-137. Representative images of migratory and invasive cells (magnification, x100) are shown. Cell migration and invasiveness was significantly decreased in miR-137-transfected cells with respect to the corresponding NC. NC, negative control; miR-137, microRNA-137.

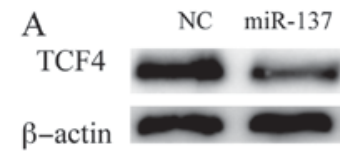

COLO205

$B$

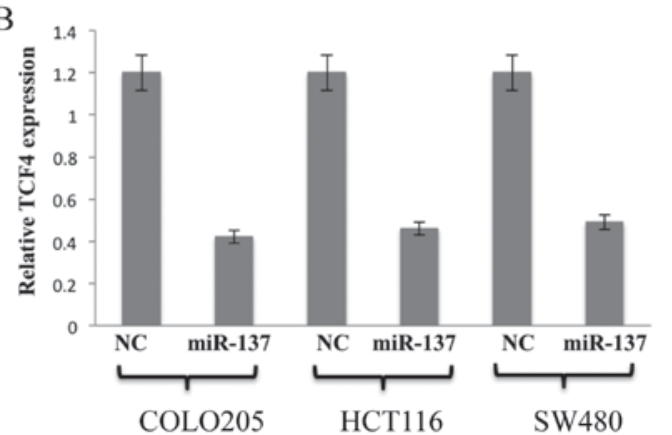

Figure 4. miR-137 negatively regulates TCF4 expression in colon cancer COLO205, HCT116 and SW480 cell lines. (A) Western blot analysis of TCF4 protein in colon cancer COLO205, HCT116 and SW480 cell lines after overexpression of miR150. Corresponding NCs and additional control ( $\beta$-actin) were used in all the experiments. (B) Reverse transcription-quantitative polymerase chain reaction analysis of TCF4 mRNA in colon cancer COLO205, HCT116 and SW480 cell lines following overexpression of miR150. Bars indicate the standard deviation. NC, negative control; miR-137, microRNA-137; TCF, transcription factor.

\section{Discussion}

In humans, miR-137 is located on the chromosome 1 p22 and undergoes transcription and extensive processing to result in a final mature miRNA 22-25 nucleotides in length. miR-137 has been associated with various types of cancer, as shown by previous studies. Studies have shown that miR-137 often functions as a tumor suppressor in gastric cancer (11), lung cancer (12), colorectal cancer (14), neuroblastoma (19), glioblastoma (20) and melanoma (21). Studies have also shown that miR-137 negatively regulates a wide spectrum of downstream targets in different types of cancers $(12,13)$; however, a minority of such direct targets has been experimentally validated. Additionally, there is a lack of data on regulatory mechanisms involving miR-137, downstream targets and cancer progression. The association between miR-137 and colorectal/colon cancer has been established in the last few years (16). One such study established that miR-137 negatively regulates $\mathrm{Cdc} 42$ expression, inducing cell cycle arrest in the G1 phase and resulting in the inhibition of invasion in colorectal cancer cells (14). Since miRNAs can target multiple transcripts, the present study identified and experimentally validated one such important target of miR-137, which may play crucial role in colon cancer pathogenesis. In the present study, TCF4 was identified as a direct target of miR-137 in COLO205, HCT116 and SW480 colon cancer cell lines. Furthermore, miR-137 suppresses the proliferation, migration and invasion of colon cancer cell lines by targeting TCF4.

The endogenous levels of miR-137 in colon cancer COLO205, HCT116 and SW480 cell lines were barely detectable. However, following the ectopic expression of miR-137, cell proliferation was significantly suppressed in all the three colon cancer cell lines. Since migration and invasion assays 
provide important insights in progression of cancer, the present study investigated the effect of miR-137 on cell migration and invasiveness. miR-137 suppressed cell migration and invasion in all the three colon cancer cell lines. These results clearly suggested that miR-137 may act as a tumor suppressor in colon cancer. There have been several well-documented studies that attribute the role of tumor suppressor function to miR-137 in various types of cancer (11-13).

The identification of the downstream targets of miR-137 was subsequently attempted to gain insights into the regulatory mechanisms of miR-137. A list of potential targets was identified by bioinformatics analysis and by adopting a 'narrow down approach'; the best scoring targets were selected. The analysis was primarily focused on one target, TCF4. There have been few studies that suggest the regulation of TCF4 by miRNAs in cancer. One such notable study was performed by Chen et al (22). This study showed that miR-24 acts an oncogene in glioma and regulates cell migration and invasion via TCF4 signaling (22). However, to the best of our knowledge, the present study is the first to identify TCF4 as a direct target of miR-137 in colon cancer.

To conclusively establish TCF4 as a direct target of miR-137, subsequent to treatment with miR-137 the levels of TCF4 were detected at the mRNA and protein level in all three colon cancer cell lines. The mRNA and protein levels of TCF4 were significantly decreased, which suggests that TCF4 is a direct target of miR-137 and miR-137 negatively regulates TCF4 in colon cancer cell lines. Since there were few other best-scoring targets predicted, it is possible that miR-137 may contribute to colon cancer progression via regulatory mechanisms using other targets.

To conclude, the present study showed that miR-137 inhibits cell proliferation, migration and invasion in colon cancer cell lines by negatively regulating the expression of TCF4.

\section{References}

1. Siegel RL, Miller KD and Jemal A: Cancer statistics, 2017. CA Cancer J Clin 67: 7-30, 2017.

2. Van Cutsem E, Nordlinger B and Cervantes A; ESMO Guidelines Working Group: Advanced colorectal cancer: ESMO Clinical Practice Guidelines for treatment. Ann Oncol 21 (Suppl 5): v93-v97, 2010.

3. Di Leva G, Garofalo M and Croce CM: MicroRNAs in cancer. Ann Rev Pathol 9: 287-314, 2014.

4. Jansson MD and Lund AH: MicroRNA and cancer. Mol Oncol 6: 590-610, 2012

5. Li M, Li J, Ding X, He M and Cheng SY: microRNA and cancer. AAPS J 12: 309-317, 2010.

6. Calin GA and Croce CM: MicroRNA signatures in human cancers. Nat Rev Cancer 6: 857-866, 2006.
7. Su J, Liang H, Yao W, Wang N, Zhang S, Yan X, Feng H, Pang W, Wang Y, Wang X, et al: MiR-143 and MiR-145 regulate IGF1R to suppress cell proliferation in colorectal cancer. PLoS One 9: e114420, 2014

8. Slaby O, Svoboda M, Fabian P, Smerdova T, Knoflickova D, Bednarikova M, Nenutil R and Vyzula R: Altered expression of miR-21, miR-31, miR-143 and miR-145 is related to clinicopathologic features of colorectal cancer. Oncology 72: 397-402, 2007.

9. Asangani IA, Rasheed SAK, Nikolova DA, Leupold JH, Colburn NH, Post S and Allgayer H: MicroRNA-21 (miR-21) post-transcriptionally downregulates tumor suppressor Pdcd4 and stimulates invasion, intravasation and metastasis in colorectal cancer. Oncogene 27: 2128-2136, 2008.

10. Motoyama K, Inoue H, Takatsuno Y, Tanaka F, Mimori K, Uetake H, Sugihara K and Mori M: Over-and under-expressed microRNAs in human colorectal cancer. Int J Oncol 34: 1069-1075, 2009.

11. Chen Q, Chen X, Zhang M, Fan Q, Luo S and Cao X: miR-137 is frequently down-regulated in gastric cancer and is a negative regulator of Cdc42. Dig Dis Sci 56: 2009-2016, 2011.

12. Zhu X, Li Y, Shen H, Li H, Long L, Hui L and Xu W: miR-137 inhibits the proliferation of lung cancer cells by targeting Cdc42 and Cdk6. FEBS Lett 587: 73-81, 2013.

13. Zhao Y, Li Y, Lou G, Zhao L, Xu Z, Zhang Y and He F: MiR-137 targets estrogen-related receptor alpha and impairs the proliferative and migratory capacity of breast cancer cells. PLoS One 7: e39102, 2012

14. Liu M, Lang N, Qiu M, Xu F, Li Q, Tang Q, Chen J, Chen X, Zhang S, Liu Z, et al: miR137 targets Cdc42 expression, induces cell cycle G1 arrest and inhibits invasion in colorectal cancer cells. Int J Cancer 128: 1269-1279, 2011.

15. Balaguer F, Link A, Lozano JJ, Cuatrecasas M, Nagasaka T, Boland CR and Goel A: Epigenetic silencing of miR-137 is an early event in colorectal carcinogenesis. Cancer Res 70: 6609-6618, 2010.

16. Gu W, Li X and Wang J: miR-139 regulates the proliferation and invasion of hepatocellular carcinoma through the WNT/TCF-4 pathway. Oncol Rep 31: 397-404, 2014.

17. Lan F, Yue X, Han L, Shi Z, Yang Y, Pu P, Yao Z and Kang C: Genome-wide identification of TCF7L2/TCF4 target miRNAs reveals a role for miR-21 in Wnt-driven epithelial cancer. Int J Oncol 40: 519-526, 2012.

18. Chen L, Zhang A, Li Y, Zhang K, Han L, Du W, Yan W, Li R, Wang Y, Wang $\mathrm{K}$, et al: MiR-24 regulates the proliferation and invasion of glioma by ST7L via $\beta$-catenin/Tcf- 4 signaling. Cancer Lett 329: 174-180, 2013.

19. Althoff K, Beckers A, Odersky A, Mestdagh P, Köster J, Bray IM, Bryan K, Vandesompele J, Speleman F, Stallings RL, et al: MiR-137 functions as a tumor suppressor in neuroblastoma by downregulating KDM1A. Int J Cancer 133: 1064-1073, 2013.

20. Chen L, Wang X, Wang H, Li Y, Yan W, Han L, Zhang K, Zhang J, Wang Y, Feng Y, et al: miR-137 is frequently down-regulated in glioblastoma and is a negative regulator of Cox-2. Eur J Cancer 48: 3104-3111, 2012.

21. Chen X, Wang J, Shen H, Lu J, Li C, Hu DN, Dong XD, Yan D and Tu L: Epigenetics, microRNAs, and carcinogenesis: Functional role of microRNA-137 in uveal melanoma. Invest Ophthalmol Vis Sci 52: 1193-1199, 2011.

22. Chen L, Han L, Zhang K, Shi Z, Zhang J, Zhang A, Wang Y, Song Y, Li Y, Jiang T, et al: VHL regulates the effects of miR-23b on glioma survival and invasion via suppression of HIF-1 $\alpha /$ VEGF and $\beta$-catenin/Tcf-4 signaling. Neuro Oncol 14: 1026-1036, 2012. 